\title{
Real Time Objects Recognition and Classification using Deep Learning Algorithm for Blind Peoples
}

\author{
K. Sivakumar ${ }^{1}$, Devi K G' ${ }^{2}$, Nikhil P C ${ }^{3}$ \\ Assistant Professor, JCT College Of Engineering And technology, Coimbatore, Tamilnadu, India ${ }^{1}$ \\ 1V CSE, JCT College Of Engineering And Technology, Coimbatore, Tamilnadu, India ${ }^{2,3}$
}

\begin{abstract}
Object detection mainly deals with identification of real- world objects such as people, animals and objects of suspense or threatening objects. Object detection algorithms use a wide range of image processing applications for extracting the objects desired portion. It is commonly used in applications such as image retrieval, security, medical defense. In this project consider as the real time object Recognition \& Classification using deep learning algorithms after that Classification objects output hear sound what categories of the object present in near we are find it. So the project will implement on PYTHON 2.7.15.
\end{abstract}

Keywords: Object Detection, Image Processing, Tracking, Deep Neural Network

\section{INTRODUCTION}

Object detection and tracking in video is a tough problem and has been drastically investigated inside the past along time. It has applications in numerous fields, consisting of video compression, video surveillance, human laptop interaction, video indexing and retrieval etc. Object detection and item tracking are two carefully related processes. The former includes locating item in the frames of a video series, the latter represents the technique of monitoring the objects spatial and temporal adjustments in everybody. Object detection can be finished through numerous procedures along with picture segmentation, history subtraction, temporal differencing, active contour fashions, and generalized transforms. In surveillance machine video sequences are typically obtained through static cameras and stuck heritage. A popular method called background subtraction is used on this state of affairs, where moving gadgets in a scene can be received by means of comparing every frame of the video with a historical past. Presently motion detection and tracking are of extremely good significance for an extensive variety of video processing packages. There are three traditional processes for items detection outlined inside the following optical glide, temporal differencing and background subtraction and many others. In this paper, our research makes a specialty of historical past reconstruction. A big range of background reconstruction techniques for constant cameras were proposed in current years: media approach, statistical approach, prediction method and assumption approach. The proposed approach additionally neutralizes the presence of shadows in the detected gadgets. There is a lot to importance to item tracking in real time environment.

\section{LITERATURE SURVEY}

“ Kalyan Kumar Hati, Pankaj Kumar Sa, and Banshidhar Majhi” an efficient Intensity Range Based Background Subtraction For Effective Object Detection is proposed. In this scheme for videos with fixed background and static cameras. The scheme suggest two different algorithms; the first one models the background from initial few frames and the second algorithm extracts the objects based on local thresholding. The strength of the scheme lies in its simplicity and the fact that, it defines an intensity range for each pixel location in the background to accommodate illumination variation as well as motion in the background. The efficiency of the scheme is shown through comparative analysis with competitive methods. Both visual as well as quantitative measures show an improved performance and the scheme has a strong potential for applications in real time survelliance. [2] D . Harihara Santhosh. P.G. Krishna Mohan an efficient "Multiple Objects Tracking Using Extended Kalman Filter, GMM and Mean Shift Algorithm" is proposed. Here the current image and the background image is differentiated by approaching conventionally in image processing. Image subtraction based algorithms are mainly used in extracting features of moving objects and take the information in frames. Here three algorithms namely Extended Kalman Filter, Gaussian Mixture Model(GMM), Mean Shift Algorithm are compared in the context of multiple object tracking. The comparative results show that GMM performs well when there are occlusions. Extended Kalman Filter fails because of abnormal behavior in the distribution 


\section{IJARCCE}

Vol. 8, Issue 2, February 2019

of random variables when there are occliions. Mean Shift Algorithm is best suitable for single object tracking and is very sensitive to window size which is adaptive. [3] Hajer Fradi Jean-Luc Dugleay an efficient “ Robust Foreground Segmentation Using Improved Gaussian Mixture Model And Optical Flow” is proposed. In this context , Gaussian Mixture Model (GMM) background subtraction has been widely employed. It is based on a probabilistic approach that achieves satisfactory performance thanks to its ability to handle complex background scenes. To achieve an improved overall performance, motion could provide a rich source of information about the scene. [4] Li Cao Yi Jiang an effective "Background Reconstruction Method For Video Objects Detection". Here the Background Subtraction is an important method to detect the moving objects, and effective background reconstructed is the key for the background subtraction. In this paper, a new background estimation method based on the PIC algorithm is proposed. Based on the background reconstructed by the improved PIC algorithm, we build a moving object detection system with Open CV. [5] Yao Yao, Yanbin Hao, Jianguo Jiang, Xueliang Liu, Richang Hong an effective technique for automatic analysis of monitoring videos is in urgent demand. As an extension of background subtraction, behavior subtraction succeeds in detecting the changes of scenes dynamics in condense hours of video stead of its photometric properties. In this paper, we first propose a new algorithm in improving behavior subtraction by maximum likelihood estimate and interval estimate methods. After that we apply the improved approach to the framework of video summarization in which the goal is to condense hours of video data into a few short segments. We finally conduct extensive experiments on real world surveillance videos.

\section{METHODOLOGY}

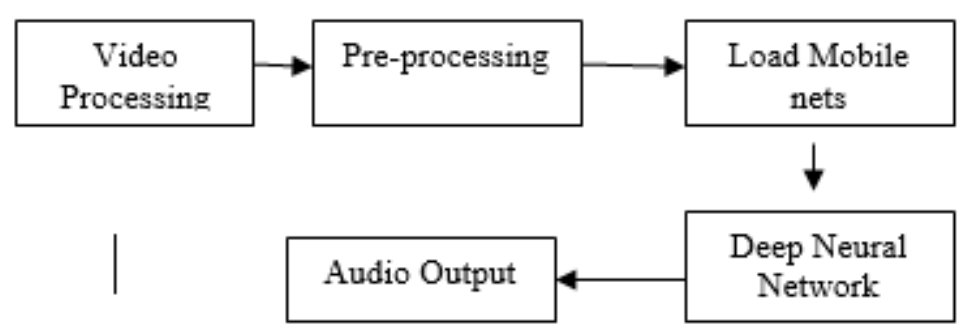

Fig (A) Block Diagram

\section{A.VIDEO PROCESSING}

In Electronics Engineering video processing is a particular case of signal processing. In particular image processing which often employs video filters and where the input and output signals are video files or video streams. Video processing is a techniques are used in television sets, DVD, video players, and other devices.

\section{B.PRE-PROCESSING}

In this image processing, preprocessing is the process of the converting color image to gray scale image. Also resize the input image such as $300 * 300$.

\section{C.GENERATE BOUNDING BOX}

In geometry, the minimum or smallest bounding or enclosing box for a point set(S) in $\mathrm{N}$ dimensions is the box with the smallest measure (area, volume or hyper volume in higher dimensions) within which all the points lie.

\section{CONDITIONS:}

- In the two dimensional case it is called the minimum bounding rectangle.

- MBRs are frequently used as an indication of the general position of a geographic feature or dataset, for either display, first-approximation spatial query, or spatial indexing purposes.

- The degree to which an "overlapping rectangles" query based on MBRs will be satisfactory (in other words, produce a low number of " false positive" hits) will depend on the extent to which individual spatial objects occupy (fill) their associated MBR.

- If the MBR is full or nearly so ( for example, a map sheet aligned with axes of latitude and longitude will normally entirely fill its associated MBR in the same coordinate space), them the "overlapping rectangles" test will be entirely reliable for that and similar spatial objects. On the other hand, if the MBR describes a dataset consisting of a diagonal line, or a small number of disjunction points ( patchy data ).

- Then the most of the MBR will be empty and an " overlapping rectangle " test will produce a high number of false positives. One system that attempts to deal with this problem, particularly for patchy data, is c- squares. 


\section{IJARCCE}

\section{International Journal of Advanced Research in Computer and Communication Engineering}

Vol. 8, Issue 2, February 2019

\section{D.LOAD MOBILE NETS}

Mobile nets are a new family of convolution neural networks that are set to blow your mind, and today we are going to train one on a custom dataset

\section{Few things that make mobile nets:}

- They are instantly small

- $\quad$ They are instantly fast

- $\quad$ They are remarkably accurate

They are easy to tune for resources Vs accuracy.

Mobile Nets are a class of convolution of neural network designed by researchers at Google . They are coined " mobilefirst" in that they are architected from the ground up to be resource - friendly and run quickly, right on your phone. Well restricted domains of object detection include face detection and pedestrian detection. Object detection has applications in many areas of computer vision, including image retrieval and video surveillance.

\section{PROPOSED SYSTEM}

\section{DEEP NEURAL NETWORK}

A Deep Neural Network (DNN) is an artificial neural network (ANN) with multiple layers between the input and output layers. Each mathematical manipulation as such as considered a layer, and complex DNN have many layers, hence the name "deep networks". Earlier versions of neural networks such as the first perceptions were shallow, composed of one input and one output layer, and at the most one hidden layers (including input and output) qualify as "deep learning". Deep learning the usage of large neural networks is teaching machines to automate the duties achieved by human visual systems. Deep getting to know has added great- human accuracy for picture class, item detection, photograph, recovery and image segmentation- even handwritten digits can be recognized. Supervised gaining knowledge of is exceptionally speedy and needs surprisingly much less computationally energy than a few other training strategies which are utilized in gadget getting to know. It has an essential downside for real - global packages, but deep gaining knowledge of networks can avoid this downside because that excellent unsupervised gaining knowledge. Deep getting to know is an aspect of Artificial Intelligence (AI) this is involved with emulating the leaning approach that human beings use to advantage sure forms of understanding. At its only, deep studying may be thought of as a way to automate predictive analytics. While traditional algorithms are linear, deep gaining knowledge of algorithm are stacked in hierarchy of growing complexity and abstraction.

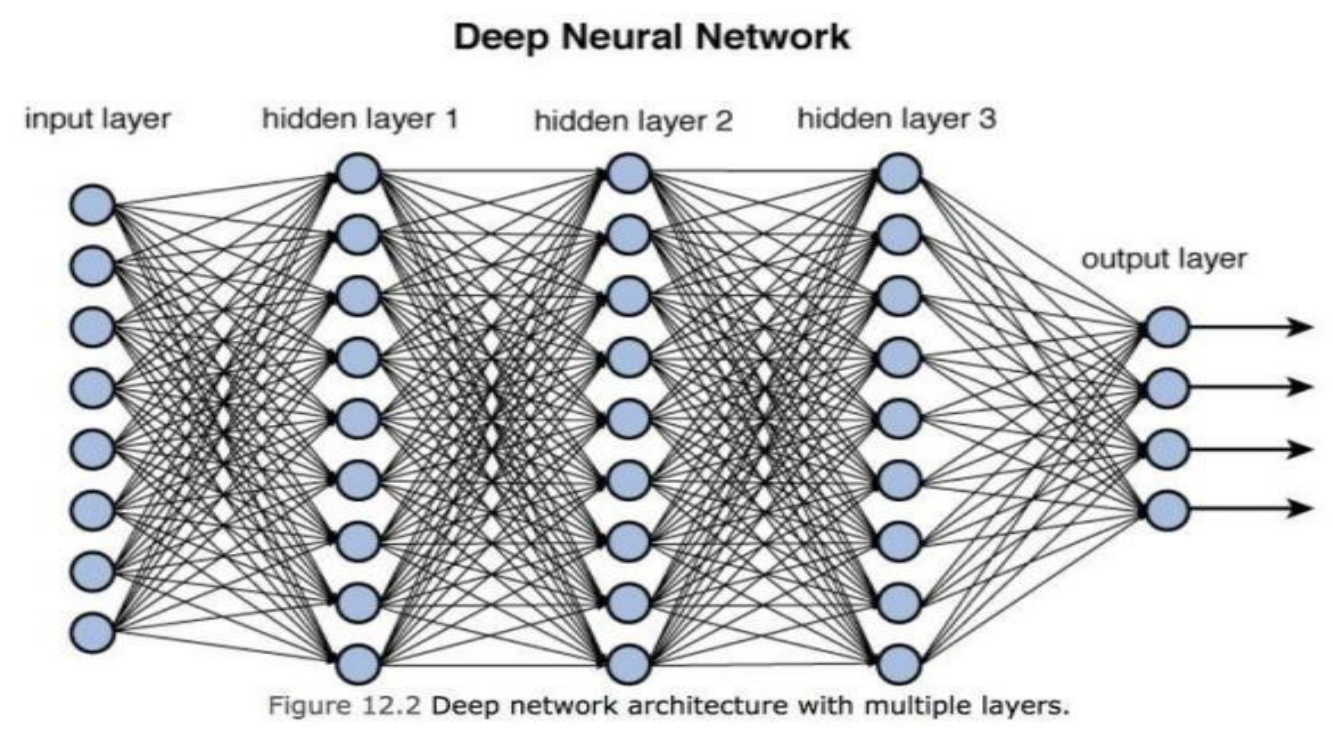

\section{FUTURE SCOPE}

This project could be further enhanced effectively more customized use. The camera should be fixed in a walking stick to sense objects and the sensed report could be made a alert to the user with the help of a linear technology such as headsets etc. this will give the technology more exposure in a personalized environment.. 


\section{IJARCCE}

Vol. 8, Issue 2, February 2019

\section{ACKNOWLEDGEMENT}

We would like to express our sincere gratitude to the staff of Department Of Computer Science and Engineering, JCT College Of Engineering And Technology

\section{CONCLUSION}

In this project implementation as the real time object Recognition \& classification using deep learning algorithms after that Classification Object output hear to the blind People to hear Sound what categories of the object present in near we are find it.

\section{REFERENCES}

[1]. H. Ye, L. Zheng and P. Liu, "Colour detection and segmentation of the scene based on Gaussian mixture model clustering," 2017 7th IEEE International Conference on Electronics Information and Emergency Communication (ICEIEC), Macau, 2017, pp. 503-506.

[2]. F. Z. Chelali, N. Cherabit and A. Djeradi, "Face recognition system using skin detection in RGB and YCbCr colour space," 2015 2nd World Symposium on Web Applications and Networking (WSWAN), Sousse, 2015, pp. 1-7.

[3]. D. H. Santosh and P. G. K. Mohan, "Multiple objects tracking using Extended Kalman Filter, GMM and Mean Shift Algorithm - A comparative study," 2014 IEEE International Conference on Advanced Communications, Control and Computing Technologies, Ramanathapuram, 2014, pp. 1484-1488

[4]. H. Fradi and J. L. Dugelay, "Robust foreground segmentation using improved Gaussian Mixture Model and optical flow," 2012 International Conference on Informatics, Electronics \& Vision (ICIEV), Dhaka, 2012, pp. 248-253

[5]. K. K. Hati, P. K. Sa and B. Majhi, "Intensity Range Based Background Subtraction for Effective Object Detection," in IEEE Signal Processing Letters, vol. 20, no. 8, pp. 759-762, Aug. 2013 